\title{
Delayed disengagement of attention from snakes in children with autism
}

\section{Tomoko Isomura, Shino Ogawa, Masahiro Shibasaki and Nobuo Masataka*}

Primate Research Institute, Kyoto University, Inuyama, Japan

\section{Edited by:}

Yusuke Moriguchi, Joetsu University of Education, Japan

Reviewed by:

Richard Gerrit Coss, University of California, Davis, USA

Shilpi Sharma, O. P. Jindal Global

University, India

\section{*Correspondence:}

Nobuo Masataka, Primate Research Institute, Kyoto University, Kanrin, Inuyama, Aichi 484-8506, Japan

e-mail: masataka.nobuo.7r@kyotou.ac.jp
In the visual search task, it is well known that detection of a tilted straight line as the target among vertical lines that act as distractors is easier than vice versa, and that detection of a snake image as the target among flower images is easier than vice versa. In this study, the degree of such search asymmetry was compared between 18 children with autism and 14 typically developing (TD) children. The results revealed that compared to TD children, children with autism were disproportionally slow when asked to detect the flower among the snake images, suggesting the possibility that they experienced difficulty of disengaging their attention from the snake images. This delayed disengagement would serve itself as an enhanced attentional bias toward snakes in children with autism that is similar to characteristics of visual search performance in anxiety patients.

Keywords: autism, snake fear, attention, cognitive impairment, biological adaptation

\section{INTRODUCTION}

Particular characteristics of childhood autism involve a profound impairment of communication (American Psychiatric Association, 2013). Typically, children with autism are known not to pay attention to things on demand, for example, when things are pointed to by caregivers, when they are called by name, or when someone enters the room (Markram and Markram, 2010 for review). It is in fact notoriously difficult to engage their attention on demand (Johnson et al., 1993). Nevertheless, they are also well known to pay abnormal and obsessive attention to detail, and to note and record their environment with exquisite clarity (Casey et al., 1993). They are capable of becoming hyper-focused and locked-in on apparently arbitrary subjects of interest, and of sustaining their attention on these subjects for unusually long periods of time. So far, these seemingly conflicting phenomena regarding the characteristics of attention impairment in autism have been interpreted as consequences of excessive on-going processing and excessive attention to endogenous domains where attention is fed back onto oneself; as a result of this internal hyper-focus, it would be more difficult for another person to command the attention of the child with autism, and it would also be more difficult for the child himself/herself to command his/her own attention voluntarily (Posner and Dehaene, 1994). This explanation is indeed confirmed by findings about the impairment in disengaging and shifting attention in children with autism (Hughes and Russell, 1993; Van Der Geest et al., 2001; Landry and Bryson, 2004; Elsabbagh et al., 2009), who on the other hand have been reported to behave comparably to typically developing (TD) children with respect to visual orienting performance per se. While all these studies were conducted by presenting biologically neutral, simple stimuli such as purely geometric shapes to the participants, the present experiment was conducted by presenting snake images as evolutionally relevant threatening stimuli (Isbell, 2009).

So far, three recent publications have shown that delayed disengagement of attention occurs in adults (Fox et al., 2002; Belopolsky et al., 2011) as well as children (Yorzinski et al., 2014; without any developmental disorder) robustly in association with complex social and biological stimuli associated with threat, but no such study has been undertaken in children with autism. Meanwhile, a series of investigations has shown that human children as well as adults have an attentional bias toward the detection of fear-relevant stimuli, such as snakes, compared to neutral stimuli, such as flowers (Öhman and Soares, 1993; Öhman and Mineka, 2001; Öhman et al., 2001; Flykt, 2005). In these studies, participants are typically presented with 3-by3 matrices of fear-relevant and neutral images. The images are presented either in black and white or in colour, and regardless of color content, reaction times (RTs) have been found to be significantly shorter for fear-relevant targets than for neutral targets. Recent studies have documented that preschool children, 8- to 14-month-old infants, and even non-human primates also detect snakes more quickly than flowers in grayscale (LoBue and DeLoache, 2008; Shibasaki and Kawai, 2009; LoBue and DeLoache, 2010; Masataka et al., 2010; Hayakawa et al., 2011).

In the present study, we hypothesized that snake images would influence the visual search performance of children with autism differently from that of TD children. Namely, when an image of a flower was presented as the target with images of snakes as distractors, children with autism would show more difficulty of detecting the target than TD children because of the slower disengagement from the threatening stimuli. If the results of the analysis of the collected data confirmed this prediction, it would indicate that the delay would associate with enhanced phobia and anxiety levels that are prominent in this developmental disorder (Markram and Markram, 2010). To evaluate the effects of such threatening stimuli, another pair of search tasks using non-biological, purely geometric items was administered; these geometric images were distinguished from one another by a feature difference along a single dimension (tilted vs. vertical straight lines). In the present 
experiments, all conditions were tested separately over a total of four blocks (each of four different types of images as a target) by requiring participants to touch the target image presented on a touch-pad.

\section{MATERIALS AND METHODS ETHICAL STATEMENTS}

This investigation was conducted according to the principles expressed in the Declaration of Helsinki. All experimental protocols are consistent with the Guide for Experimentation with Humans and were approved by the Institutional Ethics Committee of the Primate Research Institute, Kyoto University. We obtained written informed consent from the parents of all participants involved in our study.

\section{PARTICIPANTS}

Two groups of 8- to 10-year-old children participated: a group of 18 children who had been diagnosed as meeting DSM-5 diagnostic criteria for autism (American Psychiatric Association, 2013) without any anxiety or phobic symptom by psychiatrists from several hospitals in Kyoto Prefecture, Japan, and a group of 14 TD children in Japan. All children with autism and all TD children were assessed using the Autism Spectrum Quotient (Auyeung et al., 2008) at the commencement of the present study. The maximum score reported in the TD group was 17, whereas the minimum score reported in the group of children with autism was 24 . The cognitive ability of the participants was assessed using WISC III, Raven's Progressive Matrices, and Picture Vocabulary Test. Scores on each test, as well as the mean chronological age, did not differ significantly between the groups (Table $\mathbf{1}$ ).

\section{MATERIALS AND PROCEDURE}

Each participant underwent two different visual search experiments in this study. Each of the experiments again involved two search tasks. A touch-screen monitor was used for the stimulus presentation. For the first experiment, we selected 48 grayscale photographs; half of the images depicted flowers and

Table 1 | Chronological ages (years:months), WISC IQ Scores, Raven's Matrices raw scores, Picture Vocabulary Test (PVT) scores, Autism-Spectrum Quotient (ASQ) for the participants*.

\begin{tabular}{llll}
\hline Participant & $\begin{array}{l}\text { With autism } \\
(\boldsymbol{N}=\mathbf{1 8})\end{array}$ & $\begin{array}{l}\text { Control } \\
(\boldsymbol{N}=\mathbf{1 4})\end{array}$ & $\begin{array}{l}\text { Significance of } \\
\text { difference }(\boldsymbol{p})\end{array}$ \\
\hline ** \\
Age & $9: 3(0: 79)$ & $9: 3(0: 94)$ & 0.975 \\
WISC III & & & \\
Verbal IO & $101.7(16.59)$ & $103.6(17.44)$ & 0.752 \\
Performance IO & $98.1(13.59)$ & $101.8(15.90)$ & 0.475 \\
Full-Scale IO & $100.0(14.84)$ & $105.6(15.45)$ & 0.602 \\
Raven's Matrices & $29.4(4.03)$ & $29.4(4.53)$ & 0.994 \\
PVT & $52.4(11.45)$ & $52.2(11.65)$ & 0.974 \\
ASO & $29.1(5.55)$ & $11.71(4.36)$ & $<0.000$ \\
& & &
\end{tabular}

* Scores presented are mean values (SDs).

* *Probabilities were evaluated by unpaired $t$-tests $(d f=30)$. half depicted snakes. In a given trial, nine of these photographs were displayed in a 3-by-3 matrix. Each image matrix was presented on a $38.1-\mathrm{cm}$ (15-inch) screen of the monitor. Each matrix contained one target image from one category and eight distractor images from the other category. This yielded two combinations: a snake among flowers, and a flower among snakes. Each of the 24 images in the target category served as the target once. Each of the 24 pictures in the distracter category appeared eight times on average; the different distracters were presented approximately the same number of times across trials. The stimulus order was created by randomly arranging the matrices.

In both tasks of the second experiment, the stimuli consisted of two possible items that were distinguished from one another by a feature difference along a single dimension. The distinguishing feature was whether a given $0.7-\mathrm{cm}$-long straight line was tilted (rotated $18^{\circ}$ counterclockwise) or vertical. In one task, the target was a tilted line that was presented among 11 vertical line distractors. In the other task, a vertical line was the target, and the distractors were 11 tilted lines. In each task, 36 different patterns of stimuli were presented. Stimulus displays consisted of 12 elements (i.e., straight lines) arranged around an imaginary $6.2-\mathrm{cm}$ by $8.4-\mathrm{cm}$ square centerd around a fixation point. Each element measured $0.7-\mathrm{cm}$ by $0.7-\mathrm{cm}$, subtending at a visual angle of approximately $1.0^{\circ}$ horizontally and $1.0^{\circ}$ vertically. The minimum distance between the centers of each element in any display were $1.2-\mathrm{cm}$ between positions in a row and $1.2-\mathrm{cm}$ between positions in a column, and the items were presented in random locations across the screen.

In each experiment, the participant was seated in front of the monitor (approximately $40 \mathrm{~cm}$ from the base of the screen) and was told to place his/her hands on the same place at the start of each trial, making it possible to collect reliable latency data. An experimenter was seated next to the participant to monitor and instruct the child throughout the procedure.

A set of nine practice trials was completed at the beginning of the first experiment to instruct the child on how to use the touch screen. In the first three trials, a display consisting of one target (an image of a puppet) and eight distracters (images of another puppet) was presented. The participant was asked to touch the target among distracters as quickly as possible and then return his/her hands to the handprints. In the next six trials, the display consisted of one target (a snake or a flower) and eight distracters (the other), and the child was asked to touch only the target image. All images used in the practice trials were chosen randomly from the original sets of 24 .

After the participant had learned the procedure, a series of test trials was administered. The task was composed of 48 trials in the first experiment, divided into two blocks of 24 , and 72 trials in the second experiment, divided into two blocks of 36 . For each trial, a different image matrix containing one target (snake or flower, or tilted line or vertical line) and eight distracters (the remaining image type) were presented. A trial was initiated when the experimenter judged that the participant was looking at the image, to ensure that his/her full attention was on the screen before each matrix appeared. When the first block was over, another block 
began. Each participant was randomly assigned to one of two block orders.

In each trial, the RT of the participant was automatically recorded as the time between the onset of the matrix presentation and image selection. The results described in the text were solely based upon analyses of the RT data collected in this manner (RTs of incorrect responses as well as extreme RT scores-defined as values more than 2 SD above or below the mean value relative to each participant's mean RT-were excluded from the analyses).

\section{RESULTS}

The results of the experiment where snake and flower images were presented as the stimuli are shown in Figure 1. When the collected data were analyzed by a 2 (target image type, TARGET) $\times 2$ (participant group; children with autism versus TD children, CHILD) repeated measure of analysis of variance, the main effect was statistically significant for TARGET $\left[F_{(1,30)}=54.275, p<0.001\right.$, $\left.\eta_{\mathrm{p}}^{2}=0.644\right]$, but not for CHILD $\left[F_{(1,30)}=2.781, p=0.106\right.$, $\left.\eta_{\mathrm{p}}^{2}=0.085\right]$. An interaction between these factors was also significant $\left[F_{(1,30)}=7.040, p=0.013, \eta_{p}^{2}=0.190\right]$. The mean RTs (SDs) for the group of children with autism and for the control group were 1326.32 (218.36) ms and 1314.179 (247.411) ms, respectively, when they responded to a snake target, and 1961.606 (497.283) $\mathrm{ms}$ and 1613.036 (352.485) ms, respectively, when they responded to a flower target. Post hoc analyses (Bonferroni's tests) revealed that both TD children and children with autism responded to a snake target significantly more quickly than to a flower target $\left[F_{(1,30)}=9.876, p=0.004, \eta_{\mathrm{p}}^{2}=0.248\right.$ for TD children and $F_{(1,30)}=57.377, p<0.001, \eta_{\mathrm{p}}^{2}=0.657$ for children with autism]. There was no significant difference in how quickly TD children and children with autism responded to the snake targets $\left[F_{(1,30)}=0.022, p=0.884, \eta_{p}^{2}=0.001\right]$, but that children with autism responded to flower targets significantly more slowly than TD children did $\left[F_{(1,30)}=4.933, p=0.034, \eta_{\mathrm{p}}^{2}=0.141\right]$.

When vertical and tilted lines were presented, the main effect was also significant for TARGET $\left[F_{(1,30)}=49.951, p<0.001\right.$, $\left.\eta_{\mathrm{p}}^{2}=0.625\right]$, but not for CHILD $\left[F_{(1,30)}=0.445, p=0.510\right.$, $\left.\eta_{\mathrm{p}}^{2}=0.015\right]$. As shown in Figure 2, there was no significant interaction between TARGET and CHILD $\left[F_{(1,30)}=0.005, p=0.946\right.$,

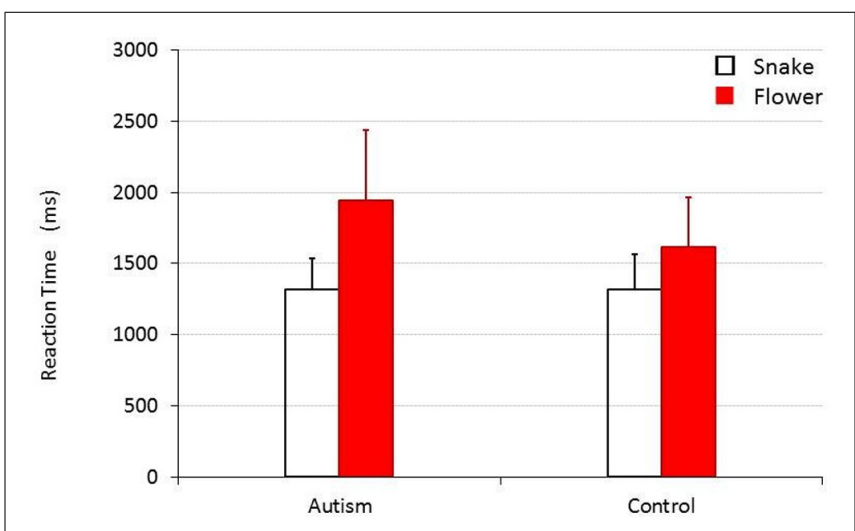

FIGURE 1 | Mean reaction time (RT) for children with and without autism to detect a snake or a flower target. Error bars represent SDs.

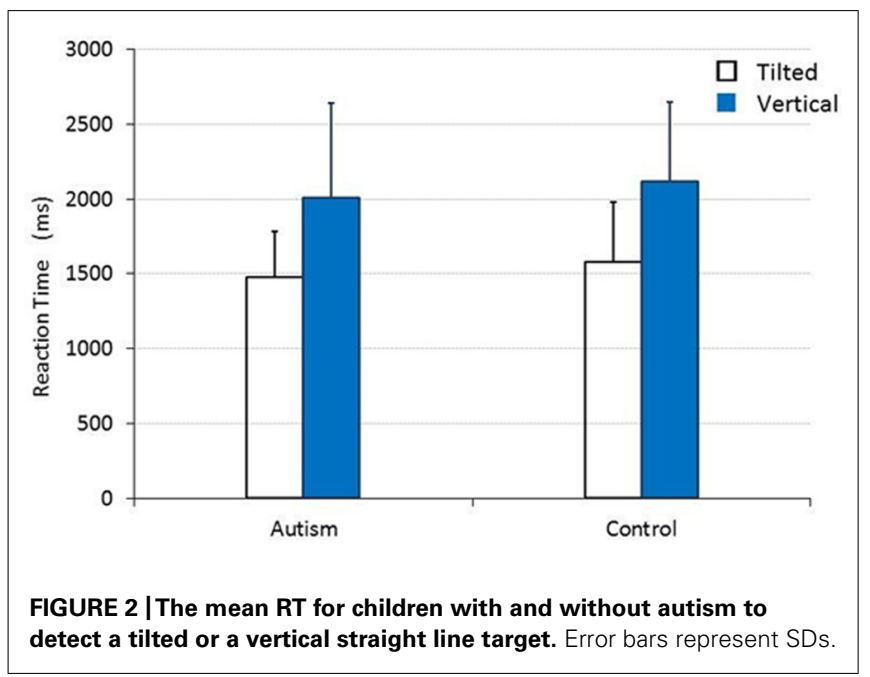

$\left.\eta_{\mathrm{p}}^{2}=0.000\right]$. The mean RTs (SDs) for the group of children with autism and for the control group of TD children were 1480.167 (305.700) $\mathrm{ms}$ and 1580.590 (401.842) ms, respectively, when they responded to a tilted line target, and 2005.947 (634.781) $\mathrm{ms}$ and 2116.654 (526.724) ms, respectively, when they responded to a vertical line target.

\section{DISCUSSION}

In this study, two different experiments were conducted, each of which involved a pair of visual search tasks. In each experiment, the same two types of stimuli were presented across tasks; in one task, one image type was designated as the target, and the other item was replicated as the distractor. In the other task, the items constituting the target and the distractor were reversed. Similar experiments reported in the past have consistently shown that detection of a tilted straight line target among vertical straight line distractors is easier than vice versa, and that detection of a snake among flower distractors is easier than vice versa. This contrasting pattern for the two related tasks is known as a "search asymmetry" effect (Treisman and Souther, 1985; Treisman and Gormican, 1988) and was again confirmed by the present study in children both with and without autism.

However, in the present study, the detection of the flower targets among snake distractors was slower in children with autism than in TD children. Although children with autism took longer to detect flowers among snakes, they performed comparably to TD children in terms of RTs on all other tasks administered (detection of a tilted line among 11 vertical lines, detection of a vertical line among 11 tilted lines and detection of a snake picture among eight flower pictures). In all, the detection of the flower among the snake images by children with autism was found to be disproportionally slow.

As an explanation for the findings, two possibilities can be suggested. One of them would be that children with autism were not necessarily faster to shift attention to snakes as threatening stimuli, but that they found it harder to disengage from the threatening images than TD children. This would be consistent with the recent argument that threat-related objects are more 
likely to delay the disengagement of attention from the threatening stimuli than to facilitate orienting the attention (Öhman and Soares, 1993; Öhman and Mineka, 2001). As an alternative explanation, one possibility would be to assume that given the finding of even longer RTs to vertical lines as distractors in TD children, they were faster in disengaging from the snakes than all other conditions, but that this reduction of the time to disengage was not observed in children with autism. A reaction pattern toward threatening objects like that observed here in TD children is that well known as "fight or flight" responses (Shibasaki and Kawai, 2009; Masataka and Shibasaki, 2012; Shibasaki et al., 2014) provided us as part of our very basic survival predispositions, and the biological structures that support the responses also usually communicate with those that provide the ability to inhibit and control reactions (e.g., "this is not a real snake, it's harmless, let's go on and search for the flower"). This reasoning would lead us to explain our results as those reflecting a lack or delay in these control processes in children with autism.

Which of these explanations is correct appears to be difficult to determine on the basis of the present findings alone. If the first explanation is correct, this delayed disengagement would, in turn, somehow relate to the formation of increased phobias, one of the most characteristic clinical features of autism, which would frequently lead to social withdrawal and avoidance (Kanner, 1943; Grandin, 1996). Indeed, similar findings to our present results have been previously observed in adult anxiety patients during visual search tasks; these patients demonstrate increased distractibility by threatening stimuli (Rinck et al., 2003, 2005; Rinck and Becker, 2005). While the present study was conducted with children with autism and without any anxiety symptoms, the possibility of this explanation could be explored by comparing performance in the snake detection experiment as conducted here between such children and children with autism and with anxiety symptoms. In order to test the possibility, it would be necessary to collect some trait anxiety data from each participant, though the current experimental literature relating to the close association of autism with such emotional disturbances is surprisingly sparse: only two recent publications have attempted to evaluate fear and anxiety levels in adults with autism using fear conditioning paradigms (Bernier et al., 2005; Gaigg and Bowler, 2007), and the responses of adults with autism were found to be virtually equivalent to those of adults without autism.

In order to examine the second possibility, it would be important to conduct visual search tasks with snake images under more controlled experimental settings; it should be noted that the present experiment was performed using a touch-pad, which certainly enabled children to perform the required task more easily than if they had used a choice-button, as in the previous research (Öhman and Soares, 1993; Öhman et al., 2001; Flykt, 2005). However, it should also be noted that the use of a touch-pad makes it difficult to isolate the cognitive cause of a difference of the performance when it is recorded. Perhaps future research could involve eye tracking with little head restraint, and even quantifying pupillary dilation during visual scanning as a measure of sympathetic arousal. Also, we still have to admit the remaining possibility that the low level visual feature could explain the observed effects because we did not match the visual feature of snake stimuli versus flower stimuli. These are clearly issues that should be investigated as a next step of the study.

\section{ACKNOWLEDGMENTS}

This research was supported by a grant (\#25285201) as well as by the Grants for Excellent Graduate Schools, from the Ministry of Education, Science, Sports and Culture, Japanese Government. We are grateful to Hiroyasu Ito, Miwa Fukushima, and Sakiko Yoshikawa for assistance in conducting experimentation and Elizabeth Nakajima and two reviewers for making comments on an earlier version of this manuscript.

\section{REFERENCES}

American Psychiatric Association. (2013). Diagnosis and Statistical Manual of Mental Disorders, 5th Edn. Washington, DC: American Psychiatric Association. doi: 10.1176/appi.books.9780890425596

Auyeung, B., Baron-Cohen, S., Wheelwright, S., and Allison, C. (2008). The autismspectrum quotient: children's version (AQ-Child). J. Autism. Dev. Disord. 38, 1230-1240. doi: 10.1007/s10803-007-0504-Z

Belopolsky, A. V., Devue, C., and Theeuwes, J. (2011). Angry faces hold the eyes. Vis. Cogn. 19, 27-36. doi: 10.1080/13506285.2010.536186

Bernier, R., Dawson, G., Panagiotides, H., and Webb, S. (2005). Individuals with autism spectrum disorders show normal responses to a fear potential startle paradigm. J. Autism. Dev. Disord. 35, 575-583. doi: 10.1007/s10803-005-0002-0

Casey, B. J., Gordon, C. T., Mannheim, G. B., and Rumsey, J. M. (1993). Dysfunctional attention in autistic savants. J. Clin. Exp. Neuropsychol. 15, 933-946. doi: 10.1080/01688639308402609

Elsabbagh, M., Volein, A., Holmboe, K., Tucker, L., Csibra, G., Baron-Cohen, S., et al. (2009). Visual orienting in the early broader autism phenotype: disengagement and facilitation. J. Child Psychol. Psychiatry 50, 637-642. doi: 10.1111/j.14697610.2008.02051.x

Flykt, A. (2005). Visual search with biological threat stimuli: accuracy, reaction times, and heart rate changes. Emotion 5, 349-353. doi: 10.1037/1528-3542.5.3.349

Fox, E., Russo, R., and Dutton, K. (2002). Attentional bias for threat: evidence for delayed disengagement from emotional faces. Cogn. Emot. 16, 355-379. doi: $10.1080 / 02699930143000527$

Gaigg, S. B., and Bowler, D. M. (2007). Differential fear conditioning in Asperger's syndrome: implications for an amygdala theory of autism. Neuropsychology 45, 2125-2134. doi: 10.1016/j.neuropsychologia.2007.01.012

Grandin, T. (1996). Thinking in Pictures. New York, NY: Vintage.

Hayakawa, S., Kawai, N., and Masataka, N. (2011). The influence of color on snake detection as visual search in human children. Sci. Rep. 1, 80. doi: $10.1038 /$ srep00080

Hughes, C., and Russell, J. (1993). Autistic children's difficulty with mental disengagement from an object: its implications for theories of autism. Dev. Psychol. 29, 498-510. doi: 10.1037/0012-1649.29.3.498

Isbell, L. A. (2009). The Fruit, the Tree, and the Serpent: Why We See So Well. Cambridge, MA: Harvard University Press.

Johnson, M. H., Posner, M. I., and Rothbart, M. K. (1993). Faciliation of saccades toward a covertly attended location in early infancy. Psychol. Sci. 5, 90-93. doi: 10.1111/j.1467-9280.1994.tb00636.x

Kanner, L. (1943). Autistic disturbances of affective contact. Nerv. Child 2, 217-250.

Landry, R., and Bryson, S. E. (2004). Impaired disengagement of attention in young children with autism. J. Child Psychol. Psychiatry 45, 1115-1122. doi: 10.1111/j.1469-7610.2004.00304.x

LoBue, V., and DeLoache, J. S. (2008). Detecting the snake in the grass - Attention to fear-relevant stimuli by adults and young children. Psychol. Sci. 19, 284-289. doi: 10.1111/j.1467-9280.2008.02081.x

LoBue, V., and DeLoache, J. S. (2010). Superior detection of threat-relevant stimuli in infancy. Dev. Sci. 13, 221-228. doi: 10.1111/j.1467-7687.2009.00872.x

Markram, K., and Markram, H. (2010). The intense world theory - A unifying theory of the neurobiology of autism. Front. Hum. Neurosci. 4:224. doi: 10.3389 /fnhum.2010.00224 
Masataka, N., Hayakawa, S., and Kawai, N. (2010). Human young children as well as adults demonstrate 'superior' rapid snake detection when typical striking posture is displayed by the snake. PLoS ONE 5:e15122. doi: 10.1371/journal.pone.0015122

Masataka, N., and Shibasaki, M. (2012). Premenstrual enhancement of snake detection in visual search in healthy women. Sci. Rep. 2, 307.

Öhman, A., Flykt, A., and Esteves, F. (2001). Emotion drives attention: detecting the snake in the grass. J. Exp. Psychol. Gen. 130, 466-478.

Öhman, A., and Mineka, S. (2001). Fears, phobias, and preparedness: toward an evolved module of fear and fear learning. Psychol. Rev. 108, 483-522.

Öhman, A., and Soares, J. J. F. (1993). On the automatic nature of phobic fear Conditioned electrodermal responses to masked fear-relevant stimuli. J. Abnorm. Psychol. 102, 121-132. doi: 10.1038/srep00307

Posner, M. I., and Dehaene, S. (1994). Attentional networks. Trend Neurosci. 17, 75-79. doi: 10.1037/0021-843X.114.2.235

Rinck, M., and Becker, E. S. (2005). A comparison of attentional biases and memory biases in women with social phobia and major depression. J. Abnorm. Psychol. 114, 62-74. doi: 10.1037/0096-3445.130.3.466

Rinck, M., Becker, E. S., Kellermann, J., and Roth, W. S. (2003). Selective attention in anxiety: distraction and enhancement in visual search? Depress. Anxiety 18, 18-28. doi: 10.1037/0033-295X.108.3.483

Rinck, M., Reinecke, A., Ellwart, T., Heuer, K., and Becker, E. S. (2005). Speeded detection and increased distraction in fear of spiders: evidence from eye movement. J. Abnorm. Psychol. 114, 235-248. doi: 10.1037/0021-843X.102.1.121

Shibasaki, M., Isomura, T., and Masataka, N. (2014). Viewing images of snakes accelerates making judgments of their colour in humans: red snake effect as an instance of 'emotional Stroop facilitation'. R. Soc. Open Sci. 1, 140066. doi: 10.1037/0021-843X.114.1.62

Shibasaki, M., and Kawai, N. (2009). Rapid detection of snakes by Japanese monkeys (Macaca fuscata): an evolutionarily predisposed visual system. J. Comp. Psychol. 123, 131-135. doi: 10.1002/da.10105
Treisman, A., and Gormican, S. (1988). Feature analysis in early vision: evidence form search aymmetries. Psychol. Rev. 95, 12-48. doi: 10.1098/rsos.140066

Treisman, A., and Souther, J. (1985). Search asymmetry: a diagnostic for preattentive processing of separable features. J. Exp. Psychol. Gen. 114, 285-310. doi: 10.1037/ a0015095

Van Der Geest, J. N., Kemner, J. N., Camfferman, G., Verbaten, M. N., and Van Engeland, H. (2001). Eye movements, visual attention, and autism: a saccadic reaction time study using the gap and overlap paradigm. Behav. Psychiatry 50, 614-619. doi: 10.1016/0166-2236(94)90078-7

Yorzinski, J. I., Penknus, M. J., Platt, M. L., and Coss, R. G. (2014). Dangerous animals capture attention in humans. Evol. Psychol. 12, 534-548. doi: 10.1037/0033295X.95.1.15

Conflict of Interest Statement: The authors declare that the research was conducted in the absence of any commercial or financial relationships that could be construed as a potential conflict of interest.

Received: 27 November 2014; accepted: 17 February 2015; published online: 03 March 2015.

Citation: Isomura T, Ogawa S, Shibasaki M and Masataka N (2015) Delayed disengagement of attention from snakes in children with autism. Front. Psychol. 6:241. doi: 10.3389/fpsyg.2015.00241

This article was submitted to Developmental Psychology, a section of the journal Frontiers in Psychology.

Copyright (C) 2015 Isomura, Ogawa, Shibasaki and Masataka. This is an open-access article distributed under the terms of the Creative Commons Attribution License (CC BY). The use, distribution or reproduction in other forums is permitted, provided the original author(s) or licensor are credited and that the original publication in this journal is cited, in accordance with accepted academic practice. No use, distribution or reproduction is permitted which does not comply with these terms. 